\title{
Cloning and characterization of an Eimeria necatrix gene encoding a gametocyte protein and associated with oocyst wall formation
}

\author{
Dandan Liu, Liqin Cao, Yulan Zhu, Changjing Deng, Shijie Su, Jinjun Xu, Wenjie Jin, Jingui Li, Lili Wu \\ and Jianping Tao*
}

\begin{abstract}
Background: Gametocyte proteins of Eimeria (E.) spp. are important components of the oocyst wall and some have been used to develop transmission-blocking vaccines against avian coccidiosis.

Methods: Total RNA isolated from E. necatrix gametocytes was utilized as templates for RT-PCR amplification and sequencing of cDNA encoding a gametocyte protein using gene-specific primers. The cDNA was cloned into the bacterial expression vector pET28a(+) and expressed in E. coli BL21 cells. The antigenicity of the recombinant gametocyte protein and its localization in different $E$. necatrix life-cycle stages were determined by western blot and indirect immunofluorescence analyses, respectively.

Results: A 731-nucleotide sequence of CDNA [GenBank: KF649255] of E. necatrix had 97.7\% identity to that of Etgam22 of E. tenella. The cDNA ORF encoded a 186-amino acid protein containing a histidine-proline-rich region. The recombinant gametocyte protein (rEnGAM22) was predominately expressed in the insoluble inclusion body and recognized by antiserum from chickens immunized with oocysts of E. necatrix, E. maxima and E. tenella. A specific antibody to the rEnGAM22 protein recognized the wall-forming bodies in macrogametocytes and the walls of oocysts and sporocysts.
\end{abstract}

Conclusions: The gene cloned from E. necatrix gametocytes is an ortholog to Etgam22 of E. tenella and presents a potential target for future recombinant subunit vaccines against coccidiosis.

Keywords: Eimeria, Gametocyte protein, Gene, Cloning and expression, Immunolocalization

\section{Background}

Coccidiosis is a parasitic disease of the intestinal tract of most domestic and wild animals and birds that is caused by protozoan parasites of the genus Eimeria. Seven Eimeria species (E. acervulina, E. brunette, E. maxima, E. mitis, E. necatrix, E. praecox, and E. tenella) are known to infect the intestinal tracts of chickens [1,2] and cause symptoms of coccidiosis, including weight loss, haemorrhagic diarrhea and death [3]. Although coccidiosis is a wellknown disease, it still remains one of the most economically important parasitic diseases of the poultry

\footnotetext{
* Correspondence: yzjptao@126.com

Jiangsu Co-innovation Center for Prevention and Control of Important

Animal Infectious Diseases and Zoonoses, Ministry of Education Key Lab for

Avian Preventive Medicine, Key Lab of Jiangsu Preventive Veterinary

Medicine, College of Veterinary Medicine, Yangzhou University, 12 East

Wenhui Road, Yangzhou, Jiangsu 225009, People's Republic of China
}

industry worldwide. In China alone, annual costs of in-feed medications or live vaccines for the control of Eimeria are estimated to cost 30-60 million US dollars [4] and the global cost is probably in the excess of $\$ 800$ million annually [5].

Prophylactic medications have been successfully used to control avian coccidiosis, but alternative strategies are needed due to the increasing emergence of drug-resistant parasites in commercial production settings [6]. The leading alternative to chemotherapeutic control is vaccination with live vaccines, which is dependent on vaccine-induced immune protection with oocysts comprising varied formulations of live wild-type or attenuated parasites of one or more species [7-9]. Moreover, there are several drawbacks to the use of live parasites, which include the need for cold storage, limited shelf-life of the vaccine, possible

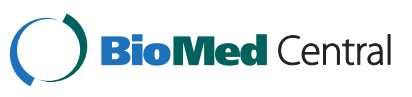


increased morbidity and mortality, and the risk of attenuated organisms reverting to a more pathogenic state. However subunit vaccines derived from intrinsic parasitic antigens or recombinant proteins from cloned DNA may overcome these difficulties [10].

Three gametocyte antigens (EmGAM56, EmGAM82, and EmGAM230) have been previously shown to play important roles in protection against E. maxima infections [11]. The subunit vaccine CoxAbix ${ }^{\ominus}$ was constructed with these proteins from purified gametocytes, and conveys transmission blocking immunity [12] that can reduce oocyst shedding. A previous field trial showed that it was at least as effective as the response from coccidiostatfed broiler controls [13]. However, the purification of the gametocyte antigens is expensive, time-consuming, and laborious, because it relies on the affinity purification of the native gametocyte antigens from parasites. Hence, a substitute vaccine based on the recombinant forms of these proteins would be advantageous and is, therefore, the focus of the current research [13-15].

E. necatrix is a highly pathogenic coccidium and can cause high mortality in susceptible birds. The first and second generation meronts of E. necatrix are primarily located within in the mid-intestinal area of host chickens and later oocyst development occurs only in the caecum [16]. Coccidiosis caused by E. necatrix mainly occurs in chickens older than 8 weeks when raised on a litter floor $[17,18]$.

Disease control relies exclusively on the protective immunity conferred to chickens. Therefore, to immunize chickens against $E$. necatrix, a planned immunization program with field isolates has been extensively implemented among breeder pullet flocks; nonetheless, such measures assumed risk of leading to outbreaks [19] and introducing pathogenic species into the environment. However, the development of subunit vaccines prepared from gametocyte antigens or recombinant proteins may overcome these difficulties. To the best of our knowledge, there are no previous reports regarding gametocyte antigens of E. necatrix and their genes. Therefore, the aim of the current study was to clone and identify a gametocyte antigen gene from E. necatrix, namely Engam22, according to cDNA sequences and localization characteristics of the recombinant protein within the gametocyte and oocyst wall, and to analyze the immunogenic characteristics of the recombinant protein.

\section{Methods}

Parasites and animals

The E. necatrix Yangzhou strain used in this study was isolated from chickens that died from $E$. necatrix infection in 2009 in Yangzhou, China, confirmed by microscopic examination and sequence analysis of the rRNA gene internal transcribed spacer (ITS) regions [20,21], and has been maintained in our laboratory. Oocysts were passaged by oral inoculation (5000 sporulated oocysts) to 3-4week-old Suqiu Yellow chickens that were purchased on the day of hatching from the Poultry Institute, Chinese Academy of Agricultural Sciences (Yangzhou, China), reared in a coccidia-free isolation facility, and allowed unlimited access to water and food that contained no anticoccidial drugs or antibiotics. Feces were collected on post-infection (PI) days 7-12, and unsporulated and sporulated oocysts were purified by centrifugation, salt flotation, and treatment with sodium hypochlorite as previously described [22].

All animal care and procedures were conducted according to the guidelines for animal use in toxicology. The study protocol was approved by the Animal Care and Use Committee of the College of Veterinary Medicine, Yangzhou University.

\section{Gametocyte preparation}

Gametocytes were isolated using previously published methods [11] with some slight modifications. Briefly, 5week-old chickens were infected with 30000 oocysts. At $168 \mathrm{~h} \mathrm{PI}$, the chickens were sacrificed and then guts removed and washed with cold SAC (1 mM phenylmethanesulfonyl fluoride, $1 \mathrm{mg} / \mathrm{mL}$ bovine serum albumin (BSA), $170 \mathrm{mM} \mathrm{NaCl}, 10 \mathrm{mM}$ Tris- $\mathrm{HCl} \mathrm{pH}$ 7, $10 \mathrm{mM}$ glucose, and $5 \mathrm{mM} \mathrm{CaCl}_{2}$ ). The caeca were cut open and the mucosal tissues removed and incubated at $37^{\circ} \mathrm{C}$ in a beaker with $0.5 \mathrm{mg} / \mathrm{mL}$ of hyaluronidase in SAC. The digested mucosal tissues were filtered through a $17-\mu \mathrm{m}$ mesh polymer filter and washed with SAC. The filtrate was then filtered through a $10-\mu \mathrm{m}$ mesh once again, and the gametocytes accumulated on this filter were washed off with SAC and centrifuged at $3000 \mathrm{rpm}$ for $5 \mathrm{~min}$ and then stored at $-80^{\circ} \mathrm{C}$ for future use.

\section{RNA extraction and amplification of the Engam 22 gene}

Total RNA was isolated from purified gametocytes using TRIzol reagent (Invitrogen, Carlsbad, CA, USA) according to manufacturer's instruction and then resuspended in diethylpyrocarbonate-treated water and was quantified using a UV spectrophotometer (NanoDrop2000; Thermo Fisher Scientific, Waltham, MA, USA) and stored at $-80^{\circ} \mathrm{C}$ for further use. The sequence of the gene coding the gametocyte protein was amplified by reverse transcription polymerase chain reaction (RT-PCR) using the RNA LA PCR Kit (TaKaRa Bio. Inc., Shiga, Japan) following the manufacturer's instruction. Specific primer sequences (Table 1, En1) were used at $0.2 \mathrm{mM}$ each to amplify the target gene under the following conditions: an initial denaturation step at $94^{\circ} \mathrm{C}$ for $4 \mathrm{~min}$; followed by 28 cycles of $94^{\circ} \mathrm{C}$ for $30 \mathrm{~s}, 56^{\circ} \mathrm{C}$ for $30 \mathrm{~s}, 72^{\circ} \mathrm{C}$ for $1.5 \mathrm{~min}$, and a final elongation step at $72^{\circ} \mathrm{C}$ for $10 \mathrm{~min}$. The PCR products were analyzed by $1.2 \%$ agarose gel electrophoresis. 
Table 1 The primers used to amplify the target gene and coding sequence of EnGAM22

\begin{tabular}{|c|c|c|}
\hline Gene ID & Forward primer $\left(5^{\prime}-3^{\prime}\right)$ & Reverse primer $\left(5^{\prime}-3^{\prime}\right)$ \\
\hline En1 & ACCCCAAAATAAAATCAAAGGC & CCATGAAGATCTCAGACGTAGC \\
\hline En2 & TCGGAATTCGACGGAGCACCTGAG & GCGAAGCTTTTAGTTGATGTCGGT \\
\hline
\end{tabular}

To confirm that the gene contained no intervening sequences, genomic DNA was isolated from purified gametocytes using the Universal Genomic DNA Extraction Kit ver. 3.0 (TaKaRa Bio., Inc.) following the manufacturer's instructions, and then used as a template for PCR under the conditions described above.

\section{Cloning, sequencing, and DNA analyses}

The RT-PCR products were purified and cloned into the TA vector pGEM-T-easy (Promega Corp., Madison, WI, USA) following the manufacturer's instructions, which were then transformed into chemically competent $\mathrm{DH} 5 \alpha$ Escherichia coli cells (Invitrogen). According to blue/white spot screening, white clones were selected for sequencing by a commercial sequencing company (Beijing Genomics Institute, Beijing, China). DNA sequences were analyzed using the BLASTN nucleotide alignment tool and predicted protein sequences were analyzed using DNAstar and the online ClustalW2 alignment tool (http://www.ebi. ac.uk/Tools/msa/clustalw2/).

\section{Recombinant protein expression and purification}

Based on the DNA sequencing results, special primers (Table 1, En2) containing EcoR I and Hind III restriction enzyme sites were designed to amplify the coding sequence of the gene excluding the signal peptide. Then, the amplicon was cloned into the bacterial expression vector pET28a(+) (Invitrogen). According to kanamycinresistance selection, recombinant plasmids were verified by sequence analysis and transformed into chemically competent E. coli BL21 cells (Invitrogen).

Recombinant protein expression from E. coli BL21 cells grown in lysogeny broth (LB) medium containing $30 \mu \mathrm{g} / \mathrm{mL}$ kanamycin at $37^{\circ} \mathrm{C}$ was induced using $1 \mathrm{mM}$ isopropyl $\beta$-D-1-thiogalactopyranoside (IPTG; Promega Corp.) at an absorbance at $600 \mathrm{~nm}$ of 0.6. The induced bacterial cells were incubated for $4 \mathrm{~h}$ and then harvested by centrifugation. The cell pellets were lysed in lysis equilibrium buffer (100 $\mathrm{mM} \mathrm{NaH}_{2} \mathrm{PO}_{4}, 10 \mathrm{mM}$ Tris-Cl, $8 \mathrm{M}$ urea, $\mathrm{pH} 8.0)$ and then sonicated $(2 \mathrm{~s} / 3 \mathrm{~s}$, for $15 \mathrm{~min})$. Next, the bacterial lysates were separated by sodium dodecyl sulfate polyacrylamide gel electrophoresis (SDS-PAGE) on $12 \%$ gels and visualized after staining with Coomassie brilliant blue. The recombinant $6 \times$ His-tagged proteins were purified from the soluble fraction of the lysate using a Ni-NTA chromatography column (GenScript, Piscataway, NJ, USA) with $500 \mathrm{mM}$ imidazole (Sigma-Aldrich, St. Louis, MO, USA). After the affinity-purified proteins were renatured in renaturation buffer $(50 \mathrm{mM}$ Tris- $\mathrm{HCl}$, $0.15 \mathrm{M} \mathrm{NaCl}, \mathrm{pH} 8.0$ ) containing 6, 4, 2, or $1 \mathrm{M}$ urea at $4^{\circ} \mathrm{C}$ for $8 \mathrm{~h}$, respectively, they were further renatured in phosphate-buffered saline (PBS; pH 8.0) at $4^{\circ} \mathrm{C}$ for $8 \mathrm{~h}$ and concentrated using polyethylene glycol (PEG8000). The yield of the purified recombinant proteins was estimated using the NanoDrop2000 spectrophotometer (Thermo Fisher Scientific) at an absorbance at $280 \mathrm{~nm}$. The purified recombinant protein (rEnGAM22) was visualized on $12 \%$ SDS-PAGE after staining with Coomassie brilliant blue, aliquoted, and stored at $-20^{\circ} \mathrm{C}$ until further use.

\section{Generation of immune sera}

Three groups of 10-day-old birds were orally inoculated with 2,000 sporulated oocysts of E. necatrix, E. tenella, and E. maxima and administered oral booster inoculations at 10 and 20 days later with of 2,500 sporulated oocysts, respectively, that were administered directly into the birds' crops using a catheter. Blood samples were collected 7 days after the second booster. Serum was separated from the blood and stored at $-80^{\circ} \mathrm{C}$ until further analyzed.

Mouse serum anti-rEnGAM22 was prepared as described previously $[23,24]$. Briefly, rEnGAM22 was diluted to $1 \mathrm{mg} / \mathrm{mL}$ in PBS and then emulsified with an equal volume of complete or incomplete Freund's adjuvant (Sigma-Aldrich). Six-week-old BALB/c mice were immunized three times at 2-week intervals with $0.1 \mathrm{mg}$ of rEnGAM22. Blood was collected 7 days after the second booster dose. Polyclonal mouse anti-rEnGAM22 antibody was separated from the blood and stored at $-80^{\circ} \mathrm{C}$ until required. Antibody levels in the mouse sera samples were determined using an indirect enzyme-linked immunosorbent assay (ELISA) as described below.

\section{Immuno-blot analysis of rEnGAM22 and gametocyte extracts}

rEnGAM22 was resolved over 12\% SDS-PAGE and transferred onto nitrocellulose membranes for $2 \mathrm{~h}$ at $100 \mathrm{~V}$ [25]. After blocking with 3\% BSA in tris-buffered saline (TBS) for $1 \mathrm{~h}$ at $37^{\circ} \mathrm{C}$, the membranes were incubated with anti- $6 \times$ His tag monoclonal antibody (dilution, 1:500; BBI Solutions, Cardiff, UK), mouse anti-rEnGAM22 polyclonal antibody (dilution, 1:100) or the convalescent chicken sera (dilution, 1:50) at room temperature for $1 \mathrm{~h}$ prior to washing three times with $0.03 \%$ Tween-20/TBS (TBST) for $10 \mathrm{~min}$, respectively. The membrane-bound antibodies were then detected using horseradish peroxidase (HRP)-conjugated rabbit anti-chicken immunoglobulin G 
(IgG; dilution, 1:1000; GenScript) or HRP-conjugated goat anti-mouse IgG (dilution, 1:5000; Kirkegaard \& Perry Laboratories, Inc. (KPL), Gathersburg, MD, USA), respectively, and developed in the presence of o-Phenylenediamine dihydrochloride (OPD) (Sigma-Aldrich) after washing five times with TBST for $10 \mathrm{~min}$, respectively. Naïve sera from chicken and mice were used as a negative controls.

To confirm that the localization of EnGAM22 in the subsequent experiment was indeed only that of EnGAM22 and not in part due to cross-reactivity with higher- weight gametocyte proteins such as GAM56- or GAM82-like proteins, the gametocyte extracts were prepared following previously published techniques [26], and were used for immunoblot analysis under the conditions described above.

\section{Preparation of tissue samples and indirect immunofluorescence analysis}

Preparation of tissue samples and indirect immunofluorescence analysis were performed as described previously $[23,24]$. Briefly, chickens were orally infected with 30,000 E. necatrix sporulated oocysts and sacrificed by $\mathrm{CO}_{2}$ inhalation and cervical dislocation at 132, 144, 156, 168, 180 and $192 \mathrm{~h} \mathrm{PI}$, respectively. The caeca and small intestines at $132 \mathrm{~h}$ PI were removed and fixed in 3\% paraformaldehyde in PBS, respectively. Fixed tissues were embedded in paraffin, and then cut into $5 \mu \mathrm{m}$ - thick sections using a microtome at room temperature. The paraffin was removed from the sections prior to inactivation of endogenous enzymes with $3 \% \mathrm{H}_{2} \mathrm{O}_{2}$ and antigen retrieval using $0.1 \%$ trypsin (Promega Corp.). After blocking overnight in $5 \%$ BSA in PBS (BSA/PBS) at $4^{\circ} \mathrm{C}$ in a humidified chamber, the sections were incubated with mouse antiEnGAM22 antibody (dilution, 1:100) in BSA/PBS for $1 \mathrm{~h}$ at $37^{\circ} \mathrm{C}$, then washed in $0.03 \%$ TWEEN-20/PBS (PBST) three times for $15 \mathrm{~min}$. Next, the sections were incubated with fluorescein isothiocyanate (FITC)-conjugate goat anti-mouse antibody (dilution, 1:100; KPL) in BSA/PBS for $1 \mathrm{~h}$ at $37^{\circ} \mathrm{C}$ and then rinsed in PBST as described above. The sections were then counter-stained with 4',6diamidino-2-phenylindole (DAPI; Roche Applied Science, Penzberg, Germany) for 5 min prior to mounting under coverslips with FluorSave Reagent (Bioworld, Consulting Laboratories, Mt. Airy, MD, USA) for visualization. Images were obtained using a Leica DM2500 reflected fluorescence microscope (Leica Microsystems GmbH, Wetzlar, Germany). Portions of the same paraffin-embedded tissue samples were also stained with hematoxylin and eosin to confirm the gametocyte and oocyst developmental processes in caecum mucosal tissues.

\section{Preparation of oocyst walls and sporocysts and indirect immunofluorescence analysis}

The purified oocyst walls and sporocysts were prepared $[24,27,28]$ and indirect immunofluorescence analyses of the same were performed as described previously [29,30]. Briefly, the sporulated oocysts $\left(1 \times 10^{7}\right)$ were washed in distilled water $\left(2,500 \mathrm{rpm}, 15 \mathrm{~min}, 4^{\circ} \mathrm{C}\right)$ three times to remove the $2 \%$ potassium dichromate storage solution and then resuspended in five volumes of PBS and sonicated with an output 3.0 , duty cycle $30 \%$ for $3 \mathrm{~s}$ intervals over 4 min in an icewater bath, until about $95 \%$ of the oocyst walls were broken. The sonicate fractions containing oocyst walls and sporocysts were centrifuged at 4,000 rpm for $10 \mathrm{~min}$ and the subsequent pellets were collected, washed with PBS for three times resuspended in 20 volumes of 1.1 M sucrose and centrifuged at 2,500 rpm for $15 \mathrm{~min}$. The top layer of fluid (sporocysts) and the pellet (oocyst walls) were collected and washed with PBS for three times, respectively. The oocyst walls and sporocysts were resuspended in methanol $\left(-20^{\circ} \mathrm{C}\right)$ and coated on glass slides, incubated for $10 \mathrm{~min}$ at $-20^{\circ} \mathrm{C}$ and washed three times with PBS for 15 min each prior to submersion in $0.1 \%$ Triton X-100 in PBS for $10 \mathrm{~min}$. After washing with PBS, the slides were blocked with $3 \%$ BSA/PBS and then stained with mouse anti-rEnGAM22 antibodies (dilution, 1:50). Lastly, the samples were labeled with tetramethyl rhodamin isothiocyanate (TRITC)-conjugated goat anti-mouse antibodies dissolved in BSA/PBS (dilution, 1:100) and imaged as described above.

\section{Results}

\section{Engam 22 isolation and sequence analysis}

The phylogenetic tree analysis of small-subunit ribosomal RNA gene showed that E. necatrix and E. tenella were most closely related among the known species of coccidia (Sporozoa) [31]. Therefore, according to the E. tenella gametocyte protein gene sequence (Etgam22; GenBank accession number: CS000361), a pair of primers (Table 1, En1) was designed to amplify the E. necatrix gametocyte protein gene of interest, Engam22. The RTPCR amplification product is depicted in Figure 1A. The sequence obtained for the Engam 22 cDNA comprised 713 base pairs (bp) (GenBank: KF649255) that included a single 561 bp open reading frame (ORF; Figure 1A), which had $97.7 \%$ identity to the 597-bp sequence of Etgam 22. The Engam22 cDNA ORF encodes a 186-amino acid (aa) polypeptide containing a histidine-proline-rich region (aa 73-176) that has been implicated in oocyst wall formation in other Eimeria species (Figure 1B). A comparison of the aa sequences of EnGAM22 and EtGAM22 via ClustalW multiple sequence alignment is shown in Figure $1 \mathrm{C}$. As shown, the aa composition of the protein deduced from Engam22, like Etgam22, is very unusual, in that only four aa [His (22.0\%), Pro (15.6\%), Gln (7.5\%) and Ala (8.6\%)], accounted for nearly $54 \%$ of all residues. EnGAM22 has a 12 aa deletion within the histidine-proline-rich region and a very high aa identity (94.7\%) to EtGAM22. Analysis using the SignalP program (www.cbs.dtu.dk/ser 


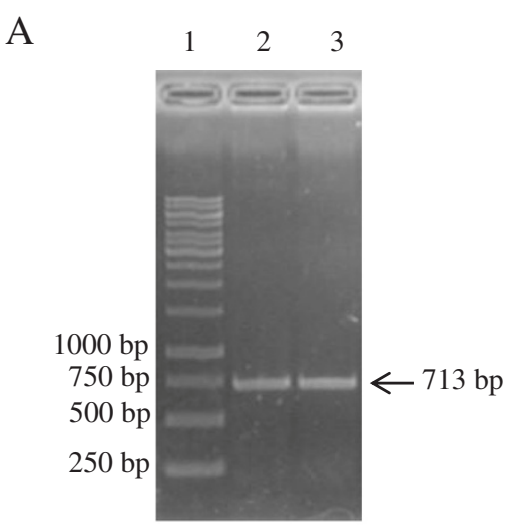

B

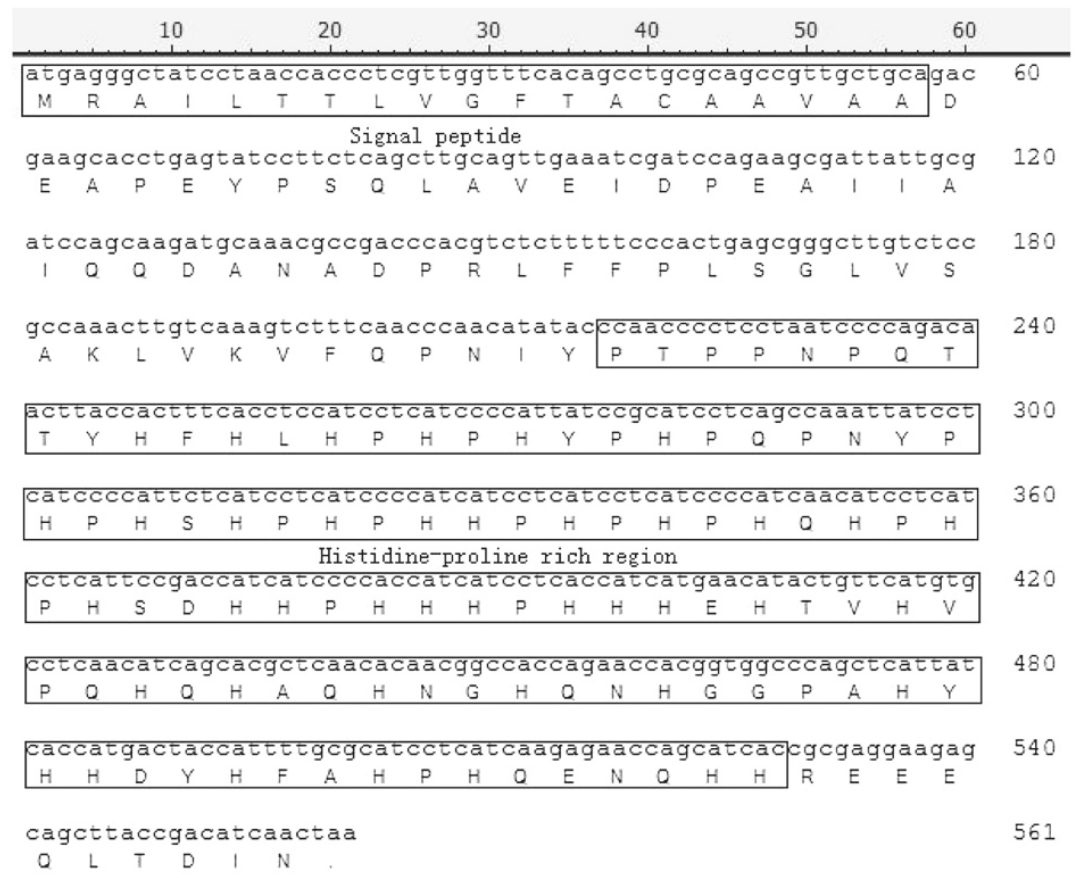

$\mathrm{C}$

Etgam22 MRTILATLVGFTACAAVAADGAPEYPSQLAVEIDPEAIIAIQQDANADPRLFFPLSGLV 60 Engam22 MRAILTTLVGFTACAAVAADEAPEYPSQLAVEIDPEAIIAIQQDANADPRLFFPLGLVS 60

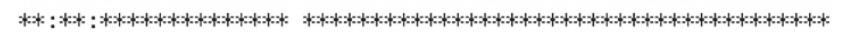

Etgam22 AKLAKVFQPNIYPTPPSPQTTYHFHLHPHPHYPHPQPNYPHPHPHHPHPHPYHPHPHPHH 120 Engam22 AKLVKVFQPNIYPTPPNPQTTYHFHLHPHPHYPHPQPNYPHPH----------SHPHPHH 110 ; $;$;

Etgam22 PHPHPHQHPHRHPDHHPHHHPHHHHHEHNWHVQHQHAQHNGHQNNGGPAHYHDYHFAH 180 Engam22 PHPHPHQHPHPHSDHHPHHHP--HHHEHTVHVPQHQHAQHNGHQNHGGP \&HYHHDYHF AH 168

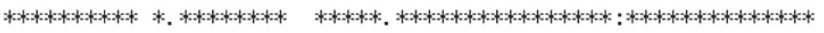

Etgam22 PHQENQHHREEEQLTDIN 198

Engam22 PHQENQHHREEEQLTDIN 186

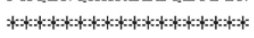

Figure 1 (See legend on next page.) 
(See figure on previous page.)

Figure 1 Sequence analysis of Engam22. (A) RT-PCR product of Engam22. Total RNA was extracted from purified gametocytes at $168 \mathrm{~h}$ PI, target gene was amplified using specific primer En1 (Table 1). Lane 1 was DNA marker, lane 2 and 3 were products of Engam22. (B) Predicted protein sequence of Engam22. The signal peptide and histidine-proline rich region was marked by box, and the splice site was between 19 and 20 amino acid. (C) Comparison of GAM22 proteins from E. tenella and E. necatrix. Invariable amino acid positions are marked with asterisks, and substitutions rated conservative and semiconservative by ClustalW are marked with colons and periods, respectively.

vices/SignalP/) revealed that the $\mathrm{N}$-terminus of EnGAM22 contained a 19-aa signal peptide (Figure 1B). The mature protein was predicted to be $21.39 \mathrm{kDa}$ with a pI of 6.73 . Comparatively, in an analysis using cDNA isolated from gametocytes, amplification of genomic DNA gave rise to a fragment of the same size, indicating that the gene contained no introns.

\section{rEnGAM22 expression and purification}

A PCR product of $\sim 522$ bp was isolated from an agarose gel and subcloned into a pET28a(+) bacterial expression vector containing the $\mathrm{NH}_{2}$ terminal $6 \times$ His tag prior to transformation into chemically competent E. coli BL21 cells. After the bacteria containing the expression vector were induced with $1 \mathrm{mM}$ IPTG for $4 \mathrm{~h}$ at $37^{\circ} \mathrm{C}$, the recombinant proteins expressed in the bacterial lysates were subjected to $12 \%$ SDS-PAGE, which revealed a protein band of $\sim 29 \mathrm{kDa}$ after staining with Coomassie brilliant blue (Figure 2A, lane 2) that had migrated less far than the expected $23.3 \mathrm{kDa}$ recombinant protein. A band of the target protein was not detected in the bacterial lysates that had not been induced with IPTG (Figure 2A, lane 3). Similarly, the protein was not detected in either the bacteria containing the wild-type vector (Figure 2A, lane 4 ) or the control bacteria (Figure 2A, lane 5). The recombinant protein was mostly insoluble (Figure 2B) and, therefore, was purified from the soluble fraction of the bacterial lysates using a Ni-NTA chromatography column. After being concentrated with PEG8000, the final protein concentration was $10 \mathrm{mg} / \mathrm{L}$.

\section{Antibody quantification by ELISA}

The ELISA protocol was essentially the same as that described previously [32]. Briefly, rEnGAM22 was diluted in $50 \mathrm{mM}$ carbonate buffer $(\mathrm{pH} 9.6)$ and $1 \mu \mathrm{g} /$ well was coated into 96-well microtiter plates, incubated at $4^{\circ} \mathrm{C}$ overnight, then washed with PBS ( $\mathrm{pH}$ 8.0) three times for $5 \mathrm{~min}$ and blocked with $1 \% \mathrm{BSA}$ for $1 \mathrm{~h}$ at $37^{\circ} \mathrm{C}$ prior to incubation with PBS-diluted mouse sera for $1 \mathrm{~h}$ at $37^{\circ} \mathrm{C}$. After washing three times for $5 \mathrm{~min}$ with PBST, HRPconjugated goat anti-mouse IgG (dilution, 1:5000; KPL) was added to each well. After incubation for $60 \mathrm{~min}$ at $37^{\circ} \mathrm{C}$ the plates were washed five times with PBST for $5 \mathrm{~min}$. Finally, immune complexes were revealed by incubating with tetramethylbenzidine (TMB, Sigma-Aldrich) and $0.3 \% \mathrm{H}_{2} \mathrm{O}_{2}$ for $10 \mathrm{~min}$. The reaction was stopped by adding $2 \mathrm{M} \mathrm{H}_{2} \mathrm{SO}_{4}$ and the absorbance values was read at
$450 \mathrm{~nm}$ using an automatic MicroELISA reader (SunriseBasic; Tecan Trading AG, Männedorf, Switzerland). All samples were run in triplicate. The results revealed a relatively high rEnGAM22-induced antibody level in the experimental mice. The optical density (OD) values were

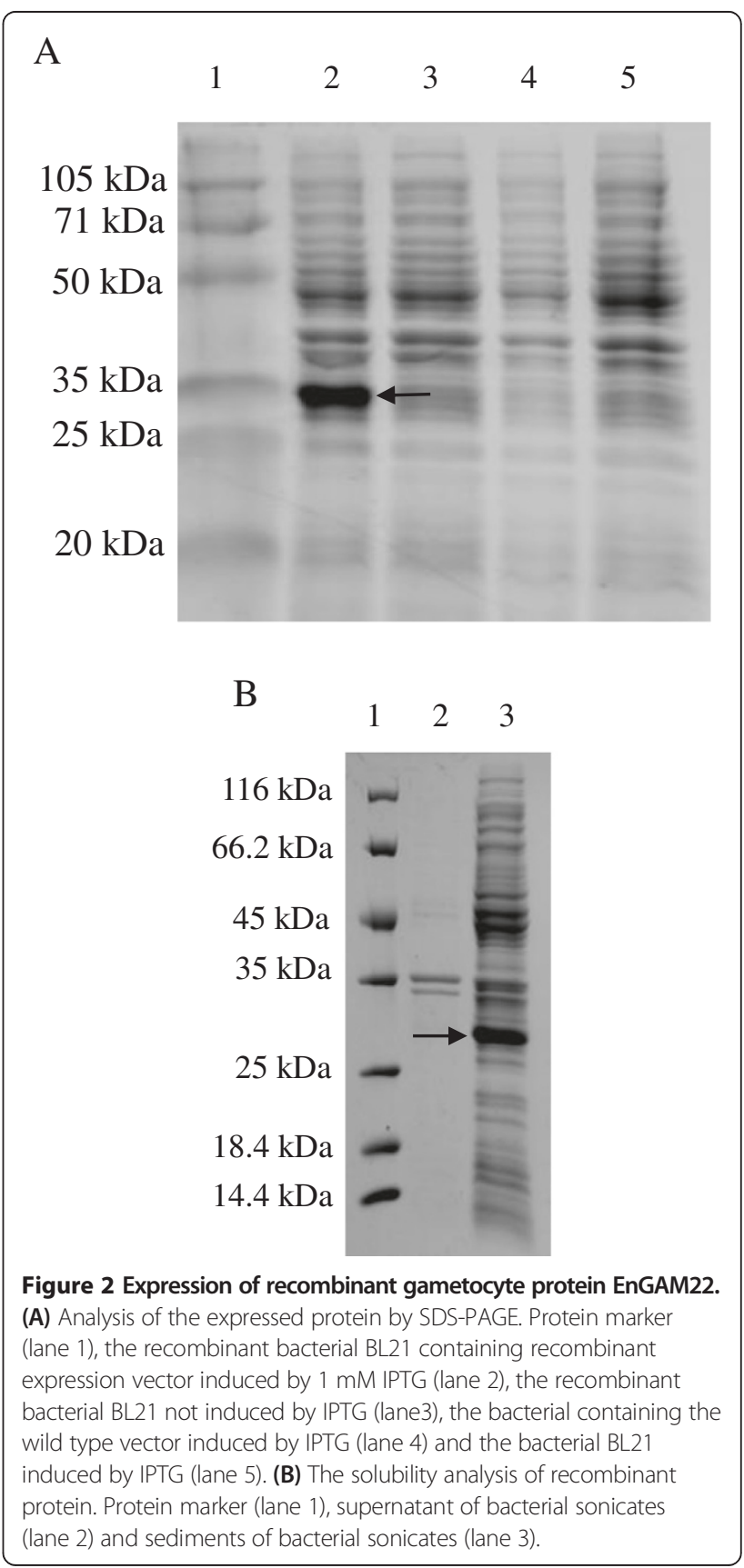


as high as 2.15 using diluted (1:200) immunized mouse serum, but only 0.15 with the control mouse serum.

\section{Immunoblot analysis of rEnGAM22 and the gametocyte extracts}

When immunoblots of the purified protein were probed using the anti- $6 \times$ His epitope tag monoclonal antibody (Figure 3A), apart from the expected $29 \mathrm{kDa}$ band, an additional $25 \mathrm{kDa}$ band was detected, which may have represented breakdown products of the full-length protein [13]. Similarly, two bands were detected using the mouse anti-rEnGAM22 antibody (Figure 3B) and the convalescent chicken sera (Figure 3C), respectively. However, among the gametocyte extracts, only a protein band of $\sim 36 \mathrm{kDa}$ was detected with the mouse anti-rEnGAM22 antibody, migrated less far than that of rEnGAM22 (Figure 3D). These
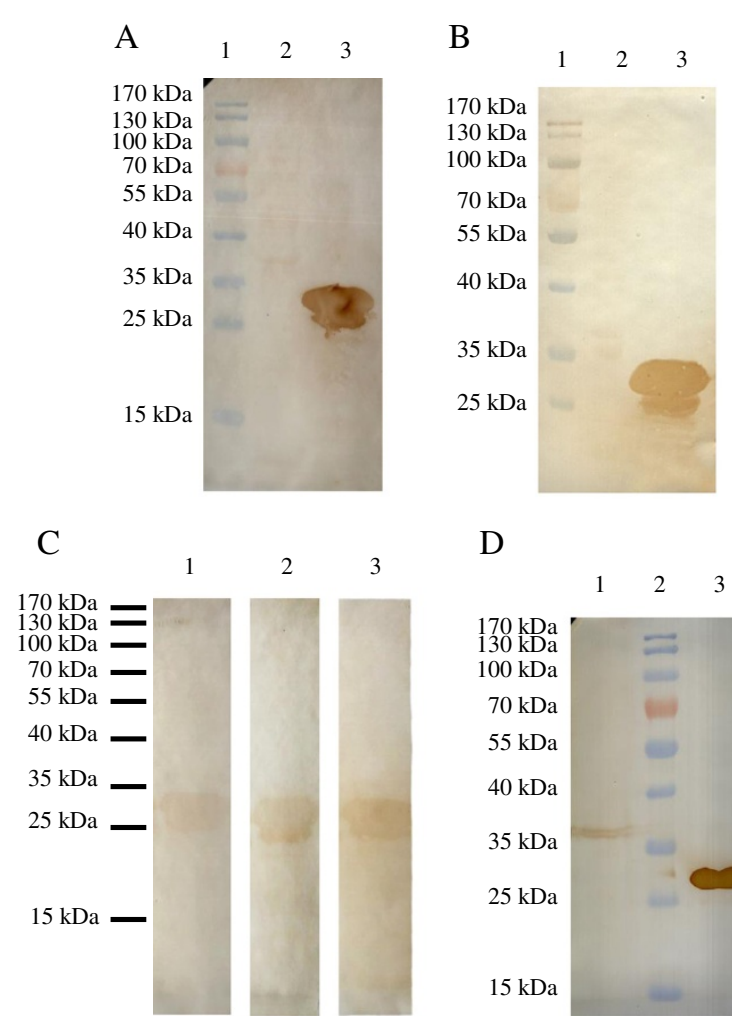

D

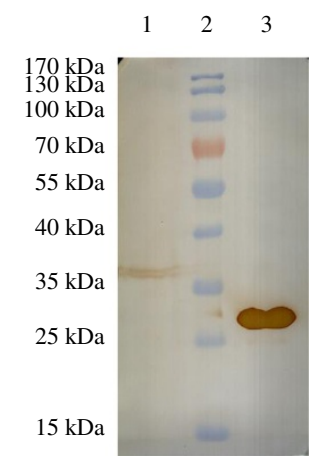

Figure 3 Western blotting analysis of rEnGAM22. The expressed protein was separated by SDS-PAGE and transferred to NC membranes, at the same time the wild type vector transferred bacterial was used as control. In (A) and (B), anti-6 $\times$ His tag monoclonal antibody (A) and mouse anti-GAM22 polyclonal antibody (B) were used as first antibody, individually. Protein marker (lane 1), the control (lane 2) and the recombinant protein (lane 3). (C) The convalescent sera from chickens were used as first antibody, anti-E. necatrix (lane 1), anti-E. tenella (lane 2) and anti-E. maxima (lane 3). (D) The gametocyte extracts were detected by the mouse anti-rEnGAM22 antibody. Gametocyte extracts (lane 1), protein marker (lane 2), and purified rEnGAM22 (lane 3). bands were not detected when the control mouse or chicken sera control samples were used (data not shown).

\section{rEnGAM22 antibodies localized to macrogametes and developing oocyst in E. necatrix}

Analysis of Engam22 revealed a 247-528 bp nucleotide sequence encoding a histidine-proline-rich region, which has been previously implicated in oocyst wall formation in E. tenella [30]. To determine whether the EnGAM22 also played a role in oocyst wall formation, we used the mouse anti-rEnGAM22 polyclonal antibody to localize the EnGAM22 during different E. necatrix life-cycles stages.

Second and third generation meronts, all stages of microgametocyte and macrogametocyte development plus oocyst formation, were observed in the haematoxylin and eosin stained tissue sections. However, parasitic development was asynchronous. At $132 \mathrm{~h} \mathrm{PI}$, the second generation of mature meronts contained a relatively high number of fully developed visible merozoites in the crypt epithelial cells retrieved from the mid-intestinal area (Figure 4A). At the same time point, the trophozoites or early gametes appeared in the epithelial cells of the caeca. At 156 h PI, a relatively large number of developing macrogametocytes were present in the lamina propria of the caeca (Figure 4B). At $168 \mathrm{~h}$ PI, most macrogametocytes developed into mature macrogametes (Figure 4C) and some exhibited formation of the first wall layer (Figure 4E) or appeared as oocysts (Figure 4F). At 192 h PI, a large number of mature oocysts were detected among the epithelial cells layers and within the cecal contents. The third generation of mature meronts was detected in all sections at 144-192 h PI (Figure 4D).

The parasites at the same developmental stage were visible in histological sections immunostained with antirEnGAM22 polyclonal mouse serum and visualized with FITC (green) and counter-stained with DAPI (blue). The anti-rEnGAM22 antibody localized to the wall forming bodies of the macrogametocytes (Figure $4 \mathrm{H}, \mathrm{I}, \mathrm{J}$ ) and the oocyst walls (Figure $4 \mathrm{~K}, \mathrm{~L}$ ). The anti-rEnGAM22 antibody seemed to be localised to the wall forming body type 2 (Figure $4 \mathrm{H}, \mathrm{J}$ ). However, the microgametes and merozoites were not recognized by the anti-rEnGAM22 antibody (Figure 4G), indicating that the EnGAM22 protein is not expressed during the schizogonic stage. In all cases, staining of the anti-rEnGAM22 polyclonal mouse serum was not observed when the tissue sections were probed with normal mouse serum.

\section{Antibodies to rEnGAM22 localized to oocyst and sporocyst walls in $E$. necatrix}

Since the oocyst walls autofluoresced blue (Figure 5), the oocyst walls and sporocysts immunostained with antirEnGAM22 antibody were visualized with TRITC in 

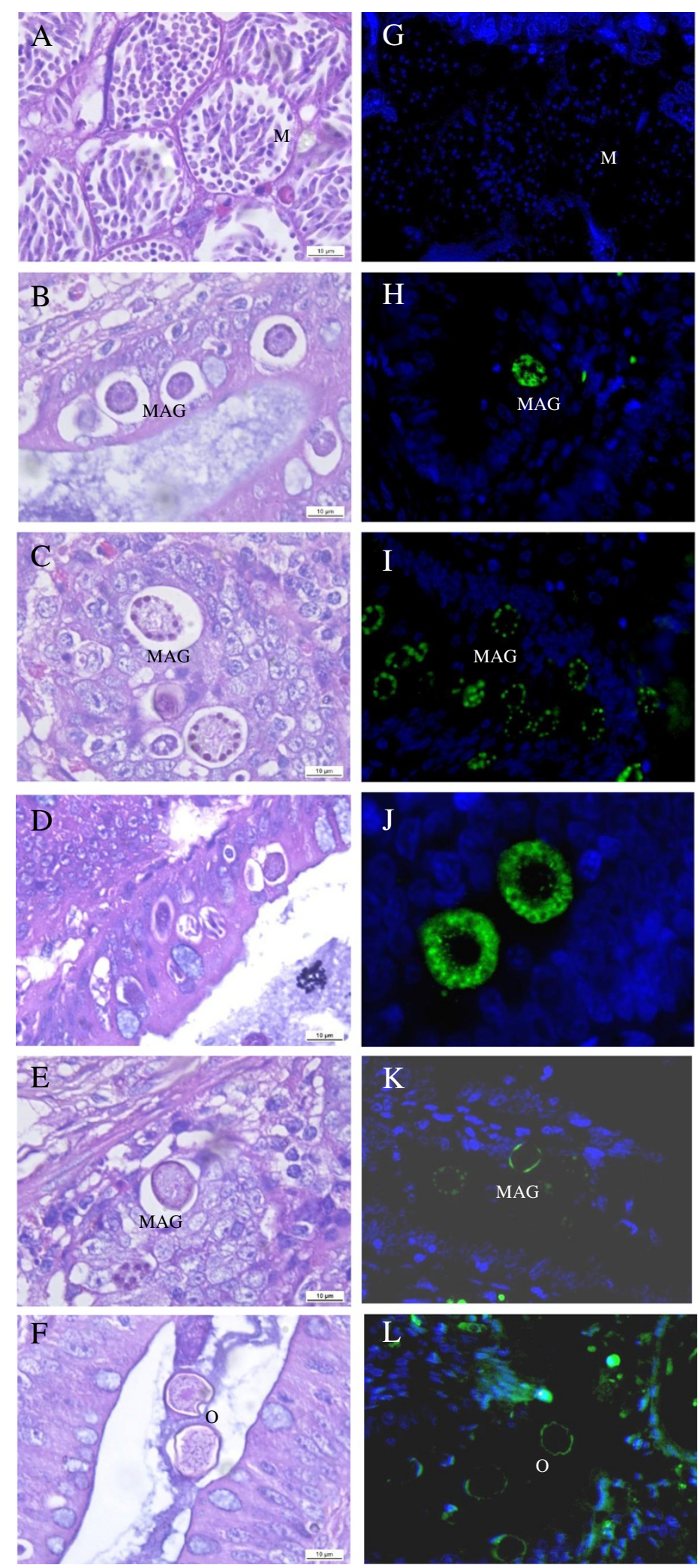

Figure 4 (See legend on next page.) 
(See figure on previous page.)

Figure 4 Localization of the anti-rEnGAM22 antibodies to the endogenous developmental stages of $E$. necatrix. The micrographs of endogenous stages of E. necatrix in histological sections, stained with H\&E (A-F) and immuno-stained with anti-rEnGAM22 and visualised with FITC (green) and counter stained with DAPI (blue) (G-L). Abbreviations: M, mature meront; MAG, macrogamont; O, oocyst. Bar represents 10 $\mu$ m, or images photographed at $\times 1000$ magnification. $(\mathbf{A}, \mathbf{G})$ Meronts with distinct merozoites in the crypt epithelial cells of the midintestinal area at $132 \mathrm{~h} \mathrm{Pl}$. (B, H) Mid-stage macrogametocytes in the crypt epithelial cells of the caeca at $156 \mathrm{~h} \mathrm{PI}$. (C, I) Mature macrogametes at $168 \mathrm{~h} \mathrm{PI}$. (J) Enlargement of macrogametes of Figure 4I. (D) The third generation mature meront at $168 \mathrm{~h}$ PI. (E, K) A macrogamete to initiate the formation of the first layer of the wall at $168 \mathrm{~h} \mathrm{PI}$. (F, L) Mature oocysts located in cecal contents at $168 \mathrm{~h} \mathrm{PI}$.

this experiment. The anti-rEnGAM22 antibodies were localized to the unsporolated oocyst walls (Figure 6A), the sporolated oocyst walls (Figure 6D), and the outer sporocyst walls (Figure 6G). However, these parasitic stages were not recognized by the negative control antibody (Figure 6C, F, I).

\section{Discussion}

The coccidian oocyst wall is a bilayered structure formed from the contents of two specific organelles, types 1 and 2 wall forming bodies (WFB1 and WFB2, respectively), found exclusively in the macrogametes stage of coccidian parasites $[33,34]$. The oocyst wall consists mainly of proteins and lipids [27,28,34]. Gametocyte proteins are precursors of the oocyst wall proteins [28,34]. To date, only a small number of genes encoding gametocyte proteins have been cloned and sequenced from avian Eimeria species, such as Emgam56 [25], Emgam82 [24], and Emgam230 (a partial sequence) [35] in E. maxima, Etgam56 (Etgam56 tmp 1), Etgam59 (Etgam56 tmp 2) and Etgam22 in E. tenella [30,36], and Eagam56 in E. acervulina (a partial sequence) [36]. Other than Etgam22, which predicts a His- and Pro-rich domain [30], all analyses of Emgam56, Etgam56, Etgam59, and Emgam82 have predicted two distinct protein domains [25,29,30,36]. Moreover, all of them are expressed specifically in gametocytes, although gametocyte-specific expression has not been detected in Etgam59 [30]. Therefore, the features of sequence characteristics and gametocyte-specific expression can be used to clone and identify the genes encoding gametocyte proteins in Eimeria species.
In the present study, we cloned a novel gene from both genomic DNA and cDNA isolated from $E$. necatrix gametocytes and found that the nucleotide sequences were comprised of 731 bp with a 561-bp ORF encoding a 186-aa protein. The protein deduced from the gene had a His- and Pro-rich domain. The antibody prepared against the recombinant protein expressed in $E$. coli BL21 cells recognized the wall-forming bodies in macrogametocytes, the walls of oocysts and sporocysts, but did not recognize either microgametes or the merozoites. In the gametocyte extracts, the anti-rEnGAM22 antibody recognized a $\sim 36 \mathrm{kDa}$ protein representing EnGAM22. These results were consistent with previous findings regarding Etgam22 [30], and further confirmed that the gene is an E. necatrix ortholog of Etgam 22 of E. tenella.

A previous study reported that both the 56 and $82 \mathrm{kDa}$ gametocyte proteins in E. maxima, with true masses of 52.45 and $62.45 \mathrm{kDa}$ [25], failed to migrate true-to-size by SDS-PAGE; particularly, the $82 \mathrm{kDa}$ protein due to its unusual aa composition [24]. Similarly, in bacterial lysates expressing the $\mathrm{r} 82$ construct, a $75 \mathrm{kDa}$ band was detected, which migrated further than that expected of a similar recombinant protein of an expected size of $67 \mathrm{kDa}$ [13]. In the present study, both the recombinant and native EnGAM22 proteins failed to migrate true-to-size by SDS-PAGE, as the bands migrated further than that expected by theoretical molecular weights. The reason for this observation was likely due to an unusual aa composition, as in EmGAM82. Additionally, in vitro translation of Etgam 22 cDNA produced
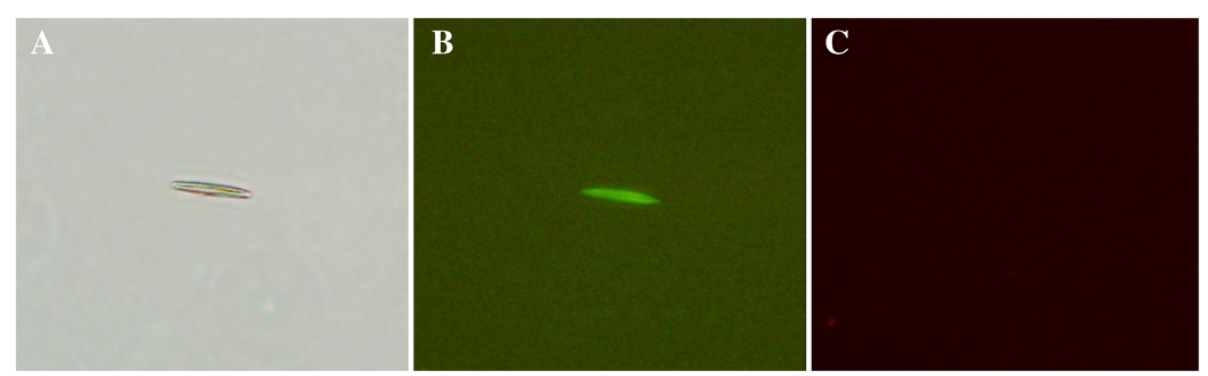

Figure 5 UV autofluorescence detection of E. necatrix oocyst walls. (A) Oocyst wall visualized under bright-field. (B) The same oocyst wall visualized under 330- to 385-nm UV light. (C) The same oocyst wall visualized under 520- to 550-nm light, which could not be detected by red fluorescence. Images photographed at $\times 1000$ magnification. 

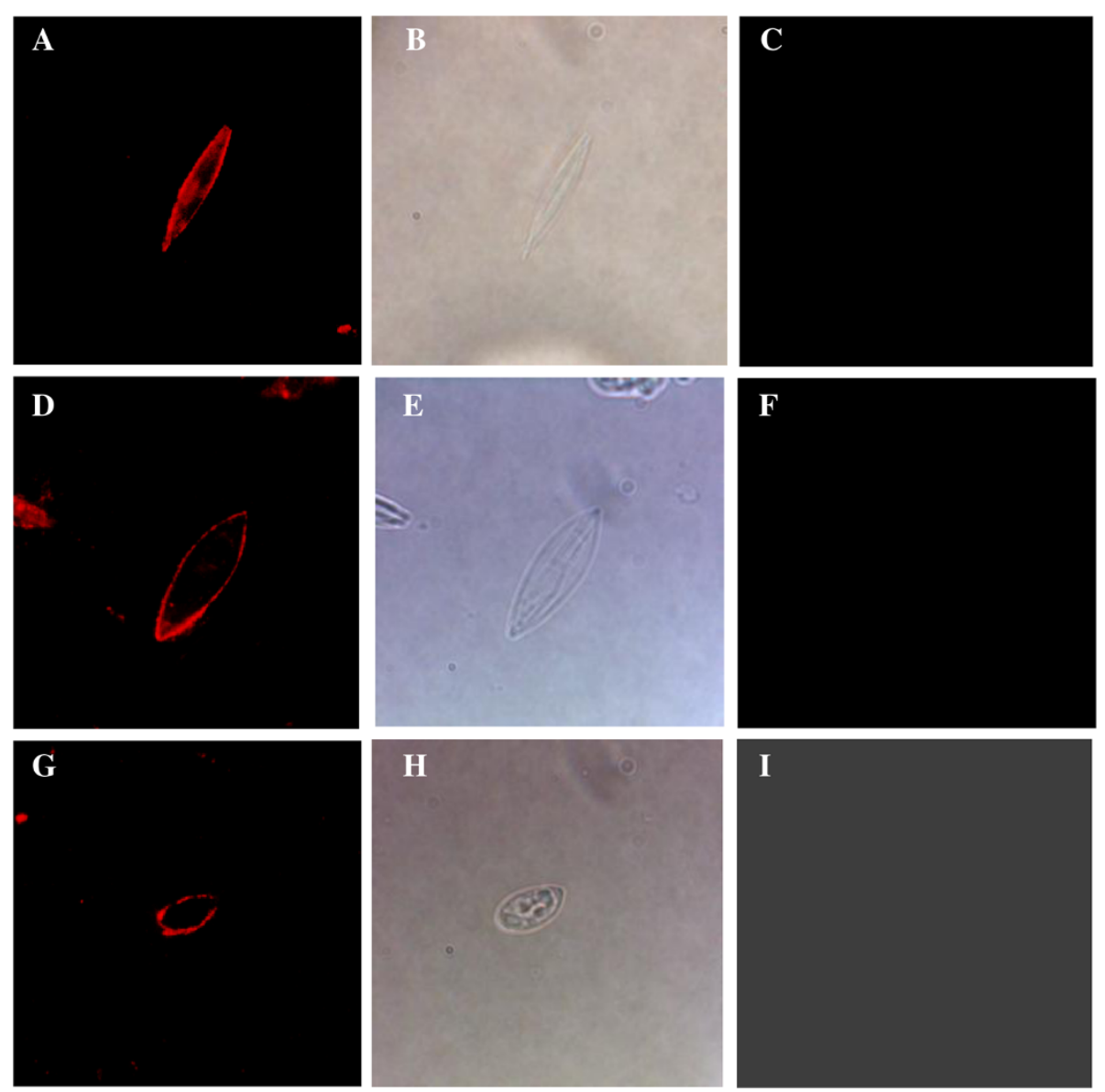

Figure 6 Anti-EnGAM22 localize to the oocyst walls and sporocyst walls of E. necatrix. Anti-EnGAM22 recognition of unsporolated oocyst walls (Panel A), sporolated oocyst walls (Panel D) and sporocyst outer walls (Panel G) was represented. Bright-field images of unsporolated oocyst walls (Panel B), sporolated oocyst walls (Panel E) and sporocyst outer walls (Panel $\mathbf{H}$ ) were also represented. As a control, the negative control antibody could not recognize unsporolated oocyst walls (Panel C), sporolated oocyst walls (Panel F) and sporocyst outer walls (Panel I). Images photographed at $\times 1000$ magnification.

a band of $\sim 25 \mathrm{kDa}$ by SDS-PAGE, which also migrated less far than that expected according to the theoretical molecular weight of $22.8 \mathrm{kDa}$ [30].

Unlike Emgam56, Etgam56, Etgam59 and Emgam82 that are single-copy intron-free genes [29,30,36,37], Etgam 22 is an intron-free multicopy gene with $\sim 12-22$ copies in head-to-tail arrangement [30]. Although Etgam 22 mRNA was readily detectable in the cecal tissue samples taken from E. tenella-infected chickens at $137 \mathrm{~h}$ PI, the EtGAM22 protein only became detectable at $168 \mathrm{~h}$ PI using the E2E5 antibody via Western blot analysis when the first unsporulated oocysts appeared in the cecum [30]. Engam22, like Etgam22, is expressed specifically at the gametocyte stage. However, the immunolocalisation results in different E. necatrix life-cycle stages revealed that Engam22 expression may occur in the mid-stage of gametocyte development (at $156 \mathrm{~h} \mathrm{PI}$ ), and EnGAM22 is transported into WFB2 prior to participation in the formation of the inner oocyst wall. The possible explanations for this difference may be due to the methods to detect the expression products. Furthermore, similar to Etgam22, Engam22 can be cloned directly from genomic DNA, implying Engam 22 is an intron-free gene. Thus, further studies are warranted to determine whether E. necatrix Engam 22 is indeed a multicopy gene.

Previous reports have confirmed that Eimeria gametocyte proteins are highly immunogenic $[11,26,38,39]$. Vaccination with gametocyte proteins of Eimeria species induces production of immunoprotective antibodies in breeding hens, which are then transferred to the developing embryos via the egg yolk, providing partial immunity to chicks upon hatching $[13,40,41]$. The anti-EmAPGA (antibody to E. maxima affinity purified gametocyte antigens) recognized proteins within the WFBs of macrogametocytes and oocyst walls of E. maxima, E. tenella and $E$. acervulina [36], which explained the features for the vaccine $\left(\mathrm{CoxAbic}{ }^{\circ}\right)$ against infections of $E$. maxima and heterologous species such E. acervulina and E. tenella [26]. 
The antibodies anti-rEmGAM56 and anti-rEmGAM82 raised against recombinant $E$. maxima gametocyte proteins, like anti-EmAPGA, reacted with various-sized proteins of gametocyte and oocyst preparations from E. maxima, E. tenella, and E. acervulina [36], in spite of only recognizing the WFB2 and inner oocyst wall via immunolocalisation analysis [33,36]. Gametocyte antigens of both E. maxima recombinant GAM56 and GAM82 were recognized by protective chicken serum raised against APGA, and can elicit a dose-dependent antibody response in chickens, suggesting that the recombinant antigens maintain the antigenic and immunogenic properties of the native proteins [13]. Furthermore, the recombinant GAM82 gametocyte antigen may stimulate the production of antigen-specific serum antibodies and a higher level of IL-2 and IL-15 mRNA, and induce protective intestinal immunity resulting in decreased oocysts shedding and reduced gut pathology [14]. In the present study, an analysis was also performed to determine whether the recombinant protein rEnGAM22 was recognized by sera from chickens that had recovered from Eimeria infection. Immunoblot, analysis showed that rEnGAM22 was recognized not only by the convalescent serum from $E$. necatrix-infected chickens, but also by that from E. tenella- and E. maxima-infected chickens, respectively. These results suggested that antigens to $E$. necatrix gametocytes, like those of E. tenella and E. maxima [41,42], might be used to develop a subunit vaccine against avian coccidiosis, and the Engam 22 gene, like Emgam56 and Emgam82, might serve as a novel candidate genes to develop a recombinant subunit vaccine.

\section{Conclusions}

In summary, we cloned a novel gene, Engam22, encoding a gametocyte protein from $E$. necatrix, which is an ortholog to Etgam 22 of E. tenella. Predictive analysis of this gene sequence revealed a His- and Pro-rich domain. The recombinant protein expressed in a bacterial expression vector had antigenic cross-reactivity to E. tenella and E. maxima. Engam22 expression begins in the early macrogametocyte and its native protein is involved in oocyst wall formation in E. necatrix.

\section{Competing interests}

The authors declare that they have no competing interests.

\section{Authors' contributions}

$J P$ and $D L$ conceived and designed the study, and critically revised the manuscript. DL, LC, YZ, CD, SS performed the experiments, analyzed the data and drafted the manuscript. JX, WJ, JL, LW helped in the study design. All authors read and approved the final manuscript.

\section{Acknowledgements}

This work was supported by the Jiangsu University and College Natural Science Foundation (11KJA230002), the Research Fund for the Doctoral Program of Higher Education of China (RFDP) (20123250110002), the University Postgraduate Research and innovation projects in 2012 of Jiangsu Province (CXZZ12-0913), the Program for Changjiang Scholars and Innovative
Research Team in University (IRT0978), and a project funded by the Priority Academic Program Development of Jiangsu Higher Education Institutions.

Received: 25 August 2013 Accepted: 2 January 2014

Published: 15 January 2014

\section{References}

1. Allen PC, Fetterer $\mathrm{RH}$ : Recent advances in biology and immunobiology of Eimeria species and in diagnosis and control of infection with these coccidian parasites of poultry. Clin Microbiol Rev 2002, 15(1):58-65.

2. Vermeulen AN, Schaap DC, Schetters TP: Control of coccidiosis in chickens by vaccination. Vet Parasitol 2001, 100(1-2):13-20.

3. Sharman PA, Smith NC, Wallach MG, Katrib M: Chasing the golden egg: vaccination against poultry coccidiosis. Parasite Immunol 2010, 32(8):590-598.

4. Hao L, Liu X, Zhou X, Li J, Suo X: Transient transfection of Eimeria tenella using yellow or red fluorescent protein as a marker. Mol Biochem Parasitol 2007, 153(2):213-215.

5. Shirley MW, Smith AL, Blake DP: Challenges in the successful control of the avian coccidia. Vaccine 2007, 25(30):5540-5547.

6. Tewari AK, Maharana BR: Control of poultry coccidiosis: changing trends. J Parasitol Dis 2011, 35(1):10-17.

7. Williams RB: Anticoccidial vaccines for broiler chickens: pathways to success. Avian Pathol 2002, 31(4):317-353.

8. Dalloul RA, Lillehoj HS: Poultry coccidiosis: recent advancements in control measures and vaccine development. Expert Rev Vaccine 2006, 5(1):143-163.

9. Lillehoj HS, Lillehoj EP: Avian coccidiosis. A review of acquired intestinal immunity and vaccination strategies. Avian Dis 2000, 44(2):408-425.

10. Jenkins MC: Advances and prospects for subunit vaccines against protozoa of veterinary importance. Vet Parasitol 2001, 101(3-4):291-310.

11. Wallach MG, Mencher D, Yarus S, Pillemer G, Halabi A, Pugatsch T: Eimeria maxima: identification of gametocyte protein antigens. Exp Parasitol 1989, 68(1):49-56.

12. Wallach M: The importance of transmission-blocking immunity in the control of infections by apicomplexan parasites. Int J Parasitol 1997, 27(10):1159-1167.

13. Belli SI, Mai K, Skene CD, Gleeson MT, Witcombe DM, Katrib M, Finger A, Wallach MG, Smith NC: Characterisation of the antigenic and immunogenic properties of bacterially expressed, sexual stage antigens of the coccidian parasite, Eimeria maxima. Vaccine 2004, 22(31-32):4316-4325.

14. Jang SI, Lillehoj HS, Lee SH, Lee KW, Park MS, Cha SR, Lillehoj EP, Subramanian BM, Sriraman R, Srinivasan VA: Eimeria maxima recombinant Gam82 gametocyte antigen vaccine protects against coccidiosis and augments humoral and cell-mediated immunity. Vaccine 2010, 28(17):2980-2985.

15. Anwar Ml, Akhtar M, Hussain I, Muhammad F, Haq AU: Effects of local gametocyte and livacox vaccines on live body weight gain and lymphoid organs in chickens. Pak Vet J 2008, 28(3):136-138.

16. Long PL: The biology of the coccidia. Baltimore, MD: University Park Press; 1982.

17. McDougald LR, Fuller AL, McMurray BL: An outbreak of Eimeria necatrix coccidiosis in breeder pullets: analysis of immediate and possible long-term effects on performance. Avian Dis 1990, 34(2):485-487.

18. Mattiello R, Boviez JD, McDougald LR: Eimeria brunetti and Eimeria necatrix in chickens of Argentina and confirmation of seven species of Eimeria. Avian Dis 2000, 44(3):711-714.

19. Onaga H, Kawahara F, Umeda K, Nagai S: Field basis evaluation of Eimeria necatrix-specific enzyme-linked immunosorbent assay (ELISA) for its utility in detecting antibodies elicited by vaccination in chickens. J Vet Med Sci 2005, 67(9):947-949.

20. Li JM, Liu DD, Wang SS, Cao LQ, Tao JP: Isolation and identification of Eimeria mitis, E. necatrix and E. praecox. In Proceedings of 11th Conference of Chinese Society of Veterinary Parasitology. Hubei, Wuhan; 2011:220. http:// www.cnki.net/kcms/detail/detail.aspx?dbcode=CPFD\&dbName=CPFD2012\& FileName $=$ ZGXJ201111001077\&v=MjMyODEq02SxuB/Qza6PRWXrCAsTSMw XFJ42CToM5KgbNH6xISq4n2bibxcp51C\%2ByyZeHT8=\&uid=WEEvREcw SIJHSIdTTGJhYkhncG1XVHdvekRla0pSbmxiY2R3dDIWMzZFVFFvVOIIMFhH THdVcHpnMnhlZFZsSA.

21. Li JM, Liu DD, Wang SS, Cao LQ, Xu JJ, Tao JP: Cloning and sequencing analyze of the internal transcribed spacer of ribosomal DNA for four 
species of Eimeria and immunological interaction between $E$. necatrix and E. maxima or E. tenella. In Proceedings of 11 th Conference of Chinese Society of Veterinary Parasitology. Hubei, Wuhan; 2011:221. http://www.cnki. net $/ \mathrm{kcms} /$ detail/detail.asp $x$ ?dbcode $=$ CPFD\&dbName $=$ CPFD2012\&FileName $=$ ZGXJ201111001077\&v=MjMyODEq02SxuB/Qza6PRWXrCAsTSMwXFJ42C ToM5KgbNH6xlSq4n2bibxcp51C\%2ByyZeHT8=\&uid=WEEvREcwSIJHSId TTGJhYkhncG1XVHdvekRlaOpSbmxiY2R3dDIWMzZFVFFvVOIIMFhHTHdVcH pnMnhIZFZsSA.

22. Wagenbach GE, Challey JR, Burns WC: A method for purifying coccidian oocysts employing clorox and sulfuric acid-dichromate solution. J Parasitol 1966, 52(6):1222.

23. Karim MJ, Basak SC, Trees AJ: Characterization and immunoprotective properties of a monoclonal antibody against the major oocyst wall protein of Eimeria tenella. Infect Immun 1996, 64(4):1227-1232.

24. Belli SI, Wallach MG, Smith NC: Cloning and characterization of the $82 \mathrm{kDa}$ tyrosine-rich sexual stage glycoprotein, GAM82, and its role in oocyst wall formation in the apicomplexan parasite, Eimeria maxima. Gene 2003, 307:201-212.

25. Belli SI, Lee M, Thebo P, Wallach MG, Schwartsburd B, Smith NC Biochemical characterisation of the 56 and $82 \mathrm{kDa}$ immunodominant gametocyte antigens from Eimeria maxima. Int J Parasitol 2002, 32(7):805-816

26. Wallach M, Smith NC, Petracca M, Miller CM, Eckert J, Braun R: Eimeria maxima gametocyte antigens: potential use in a subunit maternal vaccine against coccidiosis in chickens. Vaccine 1995, 13(4):347-354.

27. Stotish RL, Wang CC, Meyenhofer M: Structure and composition of the oocyst wall of Eimeria tenella. J Parasitol 1978, 64(6):1074-1081.

28. Mai K, Sharman PA, Walker RA, Katrib M, de Souza D, McConville MJ, Wallach MG, Belli SI, Ferguson DJ, Smith NC: Oocyst wall formation and composition in coccidian parasites. Mem Inst Oswaldo Cruz 2009, 104(2):281-289.

29. Belli SI, Wallach MG, Luxford C, Davies MJ, Smith NC: Roles of tyrosine-rich precursor glycoproteins and dityrosine- and 3,4-dihydroxyphenylalaninemediated protein cross-linking in development of the oocyst wall in the coccidian parasite Eimeria maxima. Eukaryot Cell 2003, 2(3):456-464.

30. Krucken J, Hosse RJ, Mouafo AN, Entzeroth R, Bierbaum S, Marinovski P, Hain K, Greif G, Wunderlich F: Excystation of Eimeria tenella sporozoites impaired by antibody recognizing gametocyte/oocyst antigens GAM22 and GAM56. Eukaryot Cell 2008, 7(2):202-211.

31. Morrison DA, Bornstein S, Thebo P, Wernery U, Kinne J, Mattsson JG: The current status of the small subunit rRNA phylogeny of the coccidia (Sporozoa). Int J Parasitol 2004, 34(4):501-514.

32. Meng M, He S, Zhao G, Bai Y, Zhou H, Cong H, Lu G, Zhao Q, Zhu XQ: Evaluation of protective immune responses induced by DNA vaccines encoding Toxoplasma gondii surface antigen 1 (SAG1) and 14-3-3 protein in BALB/c mice. Parasit Vectors 2012, 5:273.

33. Ferguson DJP, Belli SI, Smith NC, Wallach MG: The development of the macrogamete and oocyst wall in Eimeria maxima: immuno-light and electron microscopy. Int J Parasitol 2003, 33(12):1329-1340

34. Belli SI, Smith NC, Ferguson DJ: The coccidian oocyst: a tough nut to crack! Trend Parasitol 2006, 22(9):416-423.

35. Fried M, Mencher D, Sar-Shalom O, Wallach M: Developmental gene expression of a 230-kilodalton macrogamete-specific protein of the avian coccidial parasite, Eimeria maxima. Mol Biochem Parasit 1992, 51(2):251-262.

36. Belli SI, Ferguson DJ, Katrib M, Slapetova I, Mai K, Slapeta J, Flowers SA, Miska KB, Tomley FM, Shirley MW, et al: Conservation of proteins involved in oocyst wall formation in Eimeria maxima, Eimeria tenella and Eimeria acervulina. Int J Parasitol 2009, 39(10):1063-1070.

37. Belli SI, Witcombe D, Wallach MG, Smith NC: Functional genomics of gam56: characterisation of the role of a 56 kilodalton sexual stage antigen in oocyst wall formation in Eimeria maxima. Int J Parasitol 2002, 32(14):1727-1737.

38. Pugatsch T, Mencher D, Wallach M: Eimeria maxima: isolation of gametocytes and their immunogenicity in mice, rabbits, and chickens. Exp Parasitol 1989, 68(2):127-134.

39. Wallach M, Pillemer G, Yarus S, Halabi A, Pugatsch T, Mencher D: Passive immunization of chickens against Eimeria maxima infection with a monoclonal antibody developed against a gametocyte antigen. Infect Immun 1990, 58(2):557-562.

40. Wallach M, Halabi A, Pillemer G, Sar-Shalom O, Mencher D, Gilad M, Bendheim U, Danforth HD, Augustine PC: Maternal immunization with gametocyte antigens as a means of providing protective immunity against Eimeria maxima in chickens. Infect Immun 1992, 60(5):2036-2039.

41. Hafeez MA, Akhtar M, Javed MT, ul Haq A: Maternal immunization by egg propagated gametocyte vaccine to control Eimeria tenella infections in newly hatched chicks. Parasitol Res 2007, 100(5):1139-1141.

42. Wallach MG: The development of $\mathrm{CoxAbic}^{\oplus}$ a novel vaccine against coccidiosis. World Poultry 2002, 18:2-4.

doi:10.1186/1756-3305-7-27

Cite this article as: Liu et al: Cloning and characterization of an Eimeria necatrix gene encoding a gametocyte protein and associated with oocyst wall formation. Parasites \& Vectors 2014 7:27

\section{Submit your next manuscript to BioMed Central and take full advantage of:}

- Convenient online submission

- Thorough peer review

- No space constraints or color figure charges

- Immediate publication on acceptance

- Inclusion in PubMed, CAS, Scopus and Google Scholar

- Research which is freely available for redistribution

Submit your manuscript at www.biomedcentral.com/submit
C Biomed Central 